\title{
PSEUDO-UMBILICAL SUBMANIFOLDS OF CONSTANT CURVATURE RIEMANNIAN MANIFOLDS
}

\author{
XI-FANG CAO \\ Department of Mathematics, Yangzhou University, Yangzhou 225002, People's Republic of China \\ e-mail:xifanfcao@hotmail.com
}

(Received 5 July, 1999)

\begin{abstract}
Bai [1] gave an intrinsic integral inequality for compact minimal submanifolds of constant curvature Riemannian manifolds. In this paper, we extend Bai's result to the case of pseudo-umbilical submanifolds.
\end{abstract}

1991 Mathematics Subject Classification. 53C40, 53C42, 53C50.

1. Introduction. Let $V^{n+p}$ be an $(n+p)$-dimensional Riemannian manifold of constant curvature $a$. Let $M^{n}$ be an $n$-dimensional Riemannian manifold immersed in $V^{n+p}$. Let $h$ be the second fundamental form of the immersion, and $\xi$ the mean curvature vector. Denote by $\langle\cdot, \cdot\rangle$ the scalar product of $V^{n+p}$. If there exists a function $\lambda$ on $M^{n}$ such that

$$
\langle h(X, Y), \xi\rangle=\lambda\langle X, Y\rangle
$$

for any tangent vector $X, Y$ on $M^{n}$, then $M^{n}$ is called a pseudo-umbilical submanifold of $V^{n+p}$. It is clear that $\lambda \geq 0$. If the mean curvature vector $\xi=0$ identically, then $M^{n}$ is called a minimal submanifold of $V^{n+p}$. Every minimal submanifold of $V^{n+p}$ is itself a pseudo-umbilical submanifold of $V^{n+p}$. Z. G. Bai [1] gave an intrinsic integral inequality for compact minimal submanifolds of $V^{n+p}$ as follows.

Theorem A. Let $M^{n}$ be an n-dimensional compact minimal submanifold of $(n+p)$-dimensional constant curvature Riemannian manifold $V^{n+p}$. Then

$$
\begin{gathered}
\int_{M^{n}}\left\{p \sum R_{i j k l}^{2}+2 p \sum R_{i j}^{2}-R^{2}-n(3 p-2 n+2) a R\right\} * 1 \\
\geq n^{2}(n-1)(n-p-1) a^{2} \operatorname{Vol}\left(M^{n}\right),
\end{gathered}
$$

where $\sum R_{i j k l}^{2}$ is the square length of the Riemannian curvature tensor, $\sum R_{i j}^{2}$ is the square length of the Ricci curvature tensor and $R$ is the scalar curvature.

In this paper, we extend Theorem A to the case in which $M^{n}$ is pseudo-umbilical. We shall prove the following result.

THeOREM. Let $M^{n}$ be an $n$-dimensional compact pseudo-umbilical submanifold of $V^{n+p}$. Then 


$$
\begin{gathered}
\int_{M^{n}}\left\{p \sum R_{i j k l}^{2}+2 p \sum R_{i j}^{2}-R^{2}-n(3 p-2 n+2) a R-n(3 p-2 n) H^{2} R\right. \\
\left.+2 n^{2}(n-1) p a H^{2}+2 n^{3}(n-1) a H^{2}+n^{3} p H^{4}-n^{4} H^{4}\right\} * 1 \\
\geq n^{2}(n-1)(n-p-1) a^{2} \operatorname{Vol}\left(M^{n}\right),
\end{gathered}
$$

where $H$ is the mean curvature of $M^{n}$.

If $H \equiv 0$, i.e. $M^{n}$ is minimal, then (1.3) becomes (1.2).

2. Local formulae. In this paper, we shall make use of the following convention on the ranges of indices:

$$
1 \leq A, B, C, \cdots \leq n+p ; \quad 1 \leq i, j, k, \cdots \leq n, \quad n+1 \leq \alpha, \beta, \gamma, \cdots \leq n+p,
$$

and we shall agree that repeated indices are summed over the respective ranges.

We choose a local field of orthonormal frames $e_{1}, \cdots, e_{n+p}$ in $V^{n+p}$ such that, restricted to $M^{n}, e_{1}, \cdots, e_{n}$ are tangent to $M^{n}$. Let $\omega_{1}, \cdots, \omega_{n+p}$ be its dual frame field. Then the structure equations of $V^{n+p}$ are given by

$$
\begin{aligned}
& d \omega_{A}=-\sum \omega_{A B} \wedge \omega_{B}, \quad \omega_{A B}+\omega_{B A}=0, \\
& d \omega_{A B}=-\sum \omega_{A C} \wedge \omega_{C B}+\frac{1}{2} \sum K_{A B C D} \omega_{C} \wedge \omega_{D}, \\
& K_{A B C D}=a\left(\delta_{A C} \delta_{B D}-\delta_{A D} \delta_{B C}\right) .
\end{aligned}
$$

We restrict these forms to $M^{n}$; then we have

$$
\begin{aligned}
& \omega_{\alpha}=0, \quad \omega_{\alpha i}=\sum h_{i j}^{\alpha} \omega_{j}, \quad h_{i j}^{\alpha}=h_{j i}^{\alpha}, \\
& d \omega_{i j}=-\sum \omega_{i k} \wedge \omega_{k j}+\frac{1}{2} \sum R_{i j k l} \omega_{k} \wedge \omega_{l}, \\
& R_{i j k l}=a\left(\delta_{i k} \delta_{j l}-\delta_{i l} \delta_{j k}\right)+\sum\left(h_{i k}^{\alpha} h_{j l}^{\alpha}-h_{i l}^{\alpha} h_{j k}^{\alpha}\right), \\
& d \omega_{\alpha \beta}=-\sum \omega_{\alpha \gamma} \wedge \omega_{\gamma \beta}+\frac{1}{2} \sum R_{\alpha \beta k l} \omega_{k} \wedge \omega_{l}, \\
& R_{\alpha \beta k l}=\sum\left(h_{i k}^{\alpha} h_{i l}^{\beta}-h_{i k}^{\beta} h_{i l}^{\alpha}\right) .
\end{aligned}
$$

We call $h=\sum h_{i j}^{\alpha} \omega_{i} \omega_{j} e_{\alpha}$ the second fundamental form of the immersed manifold $M^{n}$. Denote by $S=\sum\left(h_{i j}^{\alpha}\right)^{2}$ the square length of $h, \xi=\frac{1}{n} \sum \operatorname{tr} H_{\alpha} e_{\alpha}$ the mean curvature vector and $H=\frac{1}{n} \sqrt{\sum\left(\operatorname{tr} H_{\alpha}\right)^{2}}$ the mean curvature of $M^{n}$ respectively. Here tr is the trace of the matrix $H_{\alpha}=\left(h_{i j}^{\alpha}\right)$. Now let $e_{n+1}$ be parallel to $\xi$. Then we have

$$
\operatorname{tr} H_{n+1}=n H, \quad \operatorname{tr} H_{\alpha}=0, \alpha \neq n+1 .
$$

Let $h_{i j k}^{\alpha}$ and $h_{i j k l}^{\alpha}$ denote the covariant derivative and the second covariant derivative of $h_{i j}^{\alpha}$ respectively, defined by 


$$
\begin{aligned}
& \sum h_{i j k}^{\alpha} \omega_{k}=d h_{i j}^{\alpha}-\sum h_{i k}^{\alpha} \omega_{k j}-\sum h_{j k}^{\alpha} \omega_{k i}-\sum h_{i j}^{\beta} \omega_{\beta \alpha}, \\
& \sum h_{i j k l}^{\alpha} \omega_{l}=d h_{i j k}^{\alpha}-\sum h_{i j l}^{\alpha} \omega_{l k}-\sum h_{i l k}^{\alpha} \omega_{l j}-\sum h_{l j k}^{\alpha} \omega_{l i}-\sum h_{i j k}^{\beta} \omega_{\beta \alpha} .
\end{aligned}
$$

Then we have

$$
\begin{aligned}
& h_{i j k}^{\alpha}-h_{i k j}^{\alpha}=0, \\
& h_{i j k l}^{\alpha}-h_{i j l k}^{\alpha}=\sum h_{i m}^{\alpha} R_{m j k l}+\sum h_{m j}^{\alpha} R_{m i k l}-\sum h_{i j}^{\beta} R_{\alpha \beta k l} .
\end{aligned}
$$

The Laplacian $\triangle h_{i j}^{\alpha}$ of $h_{i j}^{\alpha}$ is defined by $\triangle h_{i j}^{\alpha}=\sum h_{i j k k}^{\alpha}$. By a direct calculation we have (cf. $[2,3])$

$$
\begin{aligned}
\frac{1}{2} \triangle S & =\sum\left(h_{i j k}^{\alpha}\right)^{2}+\sum h_{i j}^{\alpha} \Delta h_{i j}^{\alpha} \\
& =\sum\left(h_{i j k}^{\alpha}\right)^{2}+\sum h_{i j}^{\alpha} h_{k k i j}^{\alpha} \\
& +\sum h_{i j}^{\alpha} h_{m k}^{\alpha} R_{m i j k}+\sum h_{i j}^{\alpha} h_{m i}^{\alpha} R_{m k j k}-\sum h_{i j}^{\alpha} h_{k i}^{\beta} R_{\alpha \beta j k} .
\end{aligned}
$$

3. Proof of Theorem. From (1.1) and (2.4) we have

$$
\left\langle h\left(e_{i}, e_{j}\right), H e_{n+1}\right\rangle=H^{2} \delta_{i j} ;
$$

therefore

$$
\sum h_{i j}^{\alpha} h_{k k i j}^{\alpha}=n H \triangle H
$$

and

$$
\sum\left(h_{i j k}^{\alpha}\right)^{2} \geq \sum\left(h_{i i k}^{n+1}\right)^{2}=n \sum\left(\nabla_{i} H\right)^{2}=n|\nabla H|^{2} .
$$

It is obvious that

$$
\frac{1}{2} \triangle H^{2}=H \triangle H+|\nabla H|^{2}
$$

and therefore

$$
\sum\left(h_{i j k}^{\alpha}\right)^{2}+\sum h_{i j}^{\alpha} h_{k k i j}^{\alpha} \geq n|\nabla H|^{2}+n H \triangle H=\frac{1}{2} n \triangle H^{2} .
$$

On the other hand, from (2.2)

$$
\begin{aligned}
\sum h_{i j}^{\alpha} h_{m k}^{\alpha} R_{m i j k} & =\frac{1}{2} \sum\left(h_{i j}^{\alpha} h_{m k}^{\alpha}-h_{m j}^{\alpha} h_{i k}^{\alpha}\right) R_{m i j k} \\
= & \frac{1}{2} \sum\left\{R_{i m j k}-a\left(\delta_{i j} \delta_{m k}-\delta_{m j} \delta_{i k}\right)\right\} R_{m i j k}=-\frac{1}{2} \sum R_{m i j k}^{2}+a R
\end{aligned}
$$

and

$$
\begin{aligned}
\sum h_{i j}^{\alpha} h_{m i}^{\alpha} R_{m k j k} & =\sum\left\{(n-1) a \delta_{m j}+n H^{2} \delta_{m j}-R_{m j}\right\} R_{m j} \\
& =-\sum R_{m j}^{2}+(n-1) a R+n H^{2} R .
\end{aligned}
$$


From (2.3) we have

$$
\sum h_{i j}^{\alpha} h_{k i}^{\beta} R_{\alpha \beta j k}=\sum h_{i j}^{\alpha} h_{l j}^{\alpha} h_{k i}^{\beta} h_{l k}^{\beta}-\sum h_{i j}^{\alpha} h_{l k}^{\alpha} h_{i k}^{\beta} h_{l j}^{\beta},
$$

while

$$
\begin{aligned}
& \sum h_{i j}^{\alpha} h_{l j}^{\alpha} h_{k i}^{\beta} h_{l k}^{\beta}=\sum\left\{(n-1) a \delta_{i l}+n H^{2} \delta_{i l}-R_{i l}\right\}^{2} \\
= & n(n-1)^{2} a^{2}-2 n^{2}(n-1) a H^{2}-2(n-1) a R-2 n H^{2} R+n^{3} H^{4}+\sum R_{i l}^{2} .
\end{aligned}
$$

Let

$$
S_{\alpha}=\sum_{i, j}\left(h_{i j}^{\alpha}\right)^{2}
$$

then we have

$$
S=\sum_{\alpha} S_{\alpha}
$$

Since

$$
S^{2}=\left(\sum_{\alpha} S_{\alpha}\right)^{2}=\sum_{\alpha} S_{\alpha}^{2}+2 \sum_{\alpha<\beta} S_{\alpha} S_{\beta}
$$

and

$$
\sum_{\alpha<\beta}\left(S_{\alpha}-S_{\beta}\right)^{2}=(p-1) \sum_{\alpha} S_{\alpha}^{2}-2 \sum_{\alpha<\beta} S_{\alpha} S_{\beta} \geq 0
$$

it follows that

$$
(p-1) \sum_{\alpha} S_{\alpha}^{2} \geq 2 \sum_{\alpha<\beta} S_{\alpha} S_{\beta}=S^{2}-\sum_{\alpha} S_{\alpha}^{2}
$$

that is

$$
\sum_{\alpha} S_{\alpha}^{2} \geq \frac{1}{p} S^{2}
$$

Since

$$
\begin{aligned}
& \sum_{i, j, l, k}\left(\sum_{\alpha} h_{i j}^{\alpha} h_{l k}^{\alpha}\right)^{2}=\sum_{i, j, l, k}\left(\sum_{\beta} h_{i k}^{\beta} h_{l j}^{\beta}\right)^{2}=\sum h_{i j}^{\alpha} h_{i j}^{\beta} h_{l k}^{\alpha} h_{l k}^{\beta} \\
= & \sum_{\alpha, \beta}\left(\sum_{i, j} h_{i j}^{\alpha} h_{i j}^{\beta}\right)^{2} \geq \sum_{\alpha}\left(\sum_{i, j} h_{i j}^{\alpha} h_{i j}^{\alpha}\right)^{2}=\sum_{\alpha} S_{\alpha}^{2},
\end{aligned}
$$


we have

$$
\begin{aligned}
& \sum h_{i j}^{\alpha} h_{l k}^{\alpha} h_{i k}^{\beta} h_{l j}^{\beta} \\
\geq & \sum h_{i j}^{\alpha} h_{l k}^{\alpha} h_{i k}^{\beta} h_{l j}^{\beta}-\frac{1}{2} \sum_{i, j, l, k}\left(\sum_{\alpha} h_{i j}^{\alpha} h_{l k}^{\alpha}\right)^{2}-\frac{1}{2} \sum_{i, j, l, k}\left(\sum_{\beta} h_{i k}^{\beta} h_{l j}^{\beta}\right)^{2}+\sum_{\alpha} S_{\alpha}^{2} \\
= & -\frac{1}{2} \sum_{i, j, l, k}\left\{\sum_{\alpha}\left(h_{i j}^{\alpha} h_{l k}^{\alpha}-h_{i k}^{\alpha} h_{l j}^{\alpha}\right)\right\}^{2}+\sum_{\alpha} S_{\alpha}^{2} \\
= & -\frac{1}{2} \sum\left\{R_{i l j k}-a\left(\delta_{i j} \delta_{l k}-\delta_{i k} \delta_{l j}\right)\right\}^{2}+\sum_{\alpha} S_{\alpha}^{2} \\
\geq & -\frac{1}{2} \sum R_{i l j k}^{2}+2 a R-n(n-1) a^{2}+\frac{1}{p} S^{2} .
\end{aligned}
$$

From (2.9), (3.5)-(3.9) we have

$$
\begin{aligned}
\frac{1}{2} \triangle S \geq & \frac{1}{2} n \triangle H^{2}-\sum R_{i j k l}^{2}-2 \sum R_{i j}^{2}+3 n a R+3 n H^{2} R \\
& -n^{2}(n-1) a^{2}-2 n^{2}(n-1) a H^{2}-n^{3} H^{4}+\frac{1}{p} S^{2} .
\end{aligned}
$$

Since $M^{n}$ is compact and

$$
S=n(n-1) a+n^{2} H^{2}-R,
$$

we have

$$
\begin{gathered}
\int_{M^{n}}\left\{p \sum R_{i j k l}^{2}+2 p \sum R_{i j}^{2}-R^{2}-n(3 p-2 n+2) a R-n(3 p-2 n) H^{2} R\right. \\
\left.+2 n^{2}(n-1) p a H^{2}+2 n^{3}(n-1) a H^{2}+n^{3} p H^{4}-n^{4} H^{4}\right\} * 1 \\
\geq n^{2}(n-1)(n-p-1) a^{2} \operatorname{Vol}\left(M^{n}\right) .
\end{gathered}
$$

Hence the Theorem is proved.

REMARK. From (2.2), if $M^{n}$ is totally geodesic, i.e. $S \equiv 0$, then (1.3) becomes the equality. It is interesting to study the geometrical property of $M^{n}$ in this case.

\section{REFERENCES}

1. Z. G. Bai, Minimal submanifolds of constant curvature Riemannian manifolds, Chinese Ann. Math. Ser. A 8 (1987), 362-367 (in Chinese).

2. S. S. Chern, M. do Carmo and S. Kobayashi, Minimal submanifolds of sphere with second fundamental form of constant length, in Functional Analysis and Related Fields (Springer-Verlag, 1970), 59-75.

3. S. T. Yau, Submanifolds with constant mean curvature, Amer. J. Math. 96 (1974), $346-366$. 